\title{
COMMENTARY
}

\section{Assessing the quantity of pulmonary edema in critically ill children}

\author{
Daniel F McAuley ${ }^{1,2 *}$, Lisa M Brown ${ }^{3,4}$ and Michael A Matthay ${ }^{4,5}$ \\ See related research by Lemson et al., http://ccforum.com/content/14/3/R105
}

\begin{abstract}
Measuring extravascular lung water may be useful for predicting outcome in adults with acute lung injury. The present commentary briefly reviews the potential role and limitations of extravascular lung water measurement in critically ill children.
\end{abstract}

In the previous issue of Critical Care, Lemson and colleagues used the single-indicator transpulmonary thermodilution method to determine the correlation between extravascular lung water (EVLW) measurement and the degree of pulmonary edema on the chest radiograph in 27 critically ill children $<10$ years old [1]. All of the children required invasive hemodynamic monitoring and mechanical ventilation. The authors report that EVLW did not correlate with the chest radiograph score of pulmonary edema or oxygenation. The authors also report, however, that EVLW is an age-dependent variable. This is an important initial step in the investigation of EVLW measurements in critically ill children.

The accumulation of fluid in the interstitium and alveolar space can be measured by quantifying EVLW. This can be measured using the single-indicator transpulmonary thermodilution method. This method has been validated against both the gold standard gravimetric method in animal models [2] and the technically more difficult and more time consuming in vivo doubleindicator technique, in which EVLW is directly measured via injection of a freely diffusible cold indicator and a plasma-bound indicator $[3,4]$. The principles underlying thermodilution have been reviewed recently $[5,6]$.

Using the transpulmonary thermodilution method, EVLW can be measured in adults at the bedside. Early

\footnotetext{
*Correspondence: d.f.mcauley@qub.ac.uk

'Regional Intensive Care Unit, Royal Victoria Hospital, Grosvenor Road, Belfast, BT12 6BA, N Ireland

Full list of author information is available at the end of the article
}

measurement of EVLW is an independent predictor for death in adult patients with acute lung injury (ALI) irrespective of etiology [7-9]. Furthermore, EVLW can be pharmacologically manipulated in both animal models [10] and in adult patients with ALI/acute respiratory distress syndrome (ARDS) [11], suggesting that EVLW may be an important measurement in patients with ALI/ ARDS. Whether EVLW is a useful measure in critically ill children, however, is unknown.

Several methodological issues in this current study should be considered. First, it is possible that measurement of EVLW in small children (age $<10$ ) is inaccurate. While the authors have previously shown that the transpulmonary thermodilution method is reliable compared with the double-indicator technique, it is possible there are technical issues common to both methods limiting the reliability of EVLW measurements in children. The authors report that EVLW measurements in adults are lower $[8,9]$. In adults with ALI, the optimal discriminatory value for EVLW indexed to predicted body weight for estimating intensive care unit mortality is $16 \mathrm{ml} / \mathrm{kg}[8,9]$. In contrast, the very high values in children in this study (median $16 \mathrm{ml} / \mathrm{kg}$ ) and other studies do not seem to have face validity for reflecting very high lung waters, especially when the degree of hypoxemia was modest $\left(\mathrm{PaO}_{2} / \mathrm{FiO}_{2}\right.$ ratio $=283 \mathrm{mmHg}$ and mean alveolar-arterial oxygen difference $=119 \mathrm{mmHg}$ ).

Technical issues with the placement of the venous catheter in the femoral site may reduce accuracy. The variability of catheter size relative to the arterial vessel may also be important. There are therefore probably technical issues in making EVLW measurements in small children - a point the authors consider in their discussion [1]. Second, several investigators measured EVLW and it would be useful to know what the coefficient of variation for EVLW measurement was in this study. It is possible that large variability in the measurements obtained in the study may confound the correlation of EVLW with chest radiograph findings and the utility of EVLW as a predictor of clinical outcomes. Third, the authors report that erroneous measurements were excluded. It would be useful if the objective criteria used to evaluate each 
measurement were reported. Finally, the authors report that EVLW was calculated afterwards. Typically the PiCCO generates the EVLW value immediately, and it is not clear what calculation was required to determine the EVLW.

One possible explanation for the lack of correlation between the measured EVLW and the chest radiograph findings is that the heterogeneity of the patients may have reduced the likelihood of detecting a correlation. Values were reported from 24 patients and the reasons for treatment in the intensive care unit were quite variable. The primary diagnosis necessitating treatment in the intensive care unit included sepsis, postoperative from cardiac or abdominal surgery and following cardiopulmonary resuscitation. Patients who are postoperative from repair of congenital heart defects may be a particularly confounding group because of abnormalities in the pulmonary blood flow in these children.

Traditionally, EVLW measurement has been indexed to actual body weight - as in this current study. Lung volumes are dependent on gender and height, however, not weight. Measurement of EVLW indexed to actual body weight underestimates this value in obese patients. Indexing EVLW to predicted body weight, which is dependent on height and gender, decreases the proportion of adult patients with ALI/ARDS with an apparently normal EVLW, and improves the predictive ability of EVLW $[8,9]$. While it is unknown whether indexing EVLW to predicted body weight is useful in children, indexing is likely to be relevant in this cohort where the range of reported weights is up to $152 \mathrm{~kg}$. EVLW was inversely correlated with height in this study; this finding supports the concept that it may be important to index EVLW to predicted body weight in children. It would also be interesting to know whether the correlation with age persists when EVLW is indexed to predicted body weight.

Finally, the ability to interpret the finding that EVLW as measured by the transpulmonary thermodilution method did not correlate with EVLW assessed using the chest radiograph may be limited for several reasons. First, the radiographic scoring system may not have accurately reflected the quantity of pulmonary edema. One important confounder can be the presence of atelectasis, which the authors addressed by assigning atelectatic lung zones with the mean radiographic score of the adjacent radiographic areas. Second, although there were two expert radiologists skilled in interpreting pediatric radiographs, the agreement between the two radiologists was only moderate, as the kappa value was 0.53 . We agree with the authors that routine daily chest radiographs do not seem to be indicated in these patients as a method for determining the extent of lung edema. A new chest radiograph scoring system has recently been validated to quantify EVLW as compared with the gravimetric method [12]. Further research is required in both adults and children to determine whether EVLW measured by the transpulmonary thermodilution method can be more accurately determined using this scoring system.

The authors are to be commended for completing this study. Overall there are many challenges in the use of this method to measure EVLW in children, which the authors acknowledge. Currently, it seems that measurement of EVLW using the transpulmonary thermodilution method routinely in clinical practice may have limited value in young children. Lemson and colleagues' study, however, raises many important questions that should prompt further research in this field in critically ill children.

\section{Abbreviations \\ ALI, acute lung injury; ARDS, acute respiratory distress syndrome; EVLW, extravascular lung water.}

\section{Competing interests}

The authors declare that they have no competing interests.

\section{Author details}

${ }^{1}$ Regional Intensive Care Unit, Royal Victoria Hospital, Grosvenor Road, Belfast, BT12 6BA, N Ireland. 'Centre for Infection and Immunity, The Queen's University of Belfast, Room 01/014, Health Sciences Building, 97 Lisburn Road, Belfast, BT9 7BL, N Ireland. ${ }^{3}$ Department of Surgery, University of California, San Francisco, 505 Parnassus Ave, San Francisco, CA 94122, California, USA. ${ }^{4}$ Cardiovascular Research Institute, University of California, San Francisco, 505 Parnassus Ave, M-917, San Francisco, CA 94143-0624, California, USA. ${ }^{5}$ Department of Medicine, Division of Pulmonary and Critical Care, and Department of Anesthesia, University of California, San Francisco, 505 Parnassus Avenue, San Francisco, CA 94143-2202, California, USA.

Published: 13 August 2010

\section{References}

1. Lemson J, van Die L, Hemelaar A, van der Hoeven J: Extravascular lung water index measurement in critically ill children does not correlate with a chest X-ray score of pulmonary edema. Crit Care 2010, 14:R105.

2. Katzenelson R, Perel A, Berkenstadt H, Preisman S, Kogan S, Sternik L, Segal E: Accuracy of transpulmonary thermodilution versus gravimetric measurement of extravascular lung water. Crit Care Med 2004, 32:1550-1554.

3. Neumann P: Extravascular lung water and intrathoracic blood volume: double versus single indicator dilution technique. Intensive Care Med 1999, 25:216-219.

4. Lemson J, Backx AP, van Oort AM, Bouw TPWJM, van der Hoeven JG: Extravascular lung water measurement using transpulmonary thermodilution in children. Pediatr Crit Care Med 2009, 10:227-233.

5. Effros RM, Pornsuriyasak P, Porszasz J, Casaburi R: Indicator dilution measurements of extravascular lung water: basic assumptions and observations. Am J Physiol Lung Cell Mol Physiol 2008, 294:L1023-L1031.

6. Brown LM, Liu KD, Matthay MA: Measurement of extravascular lung water using the single indicator method in patients: research and potential clinical value. Am J Physiol Lung Cell Mol Physiol 2009, 297:L547-L558.

7. Matthay MA: Measurement of extravascular lung water in patients with pulmonary edema. Am J Physiol Lung Cell Mol Physiol 2008, 294:L1021-L1022.

8. Craig TR, Duffy MJ, Shyamsundar M, McDowell C, McLaughlin B, Elborn JS, McAuley DF: Extravascular lung water indexed to predicted body weight is a novel predictor of intensive care unit mortality in patients with acute lung injury. Crit Care Med 2010, 38:114-120.

9. Phillips $C R$, Chesnutt MS, Smith SM: Extravascular lung water in sepsisassociated acute respiratory distress syndrome: indexing with predicted body weight improves correlation with severity of illness and survival. Crit Care Med 2008, 36:69-73.

10. McAuley DF, Frank JA, Fang $X$, Matthay MA: Clinically relevant concentrations of $\beta_{2}$-adrenergic agonists stimulate maximal cyclic 
adenosine monophosphate-dependent airspace fluid clearance and decrease pulmonary edema in experimental acid-induced lung injury. Crit Care Med 2004, 32:1470-1476.

11. Perkins GD, McAuley DF, Thickett DR, Gao F: The $\beta$-agonist lung injury trial (BALTI): a randomized placebo-controlled clinical trial. Am J Respir Crit Care Med 2006, 173:281-287.

12. Neyrinck AP, O'Neal H, Lee JW, Curtis B, Calfee CS, Matthay MA, Ware LB: Quantification of pulmonary edema on chest radiograph compared with direct measurements of extravascular lung water in humans. Am J Respir Crit Care Med 2009, 179:A3385.

doi:10.1186/cc9199

Cite this article as: McAuley DF, et al:: Assessing the quantity of pulmonary edema in critically ill children. Critical Care 2010, 14:189. 